\title{
Helical Turing patterns in the Lengyel-Epstein model in thin cylindrical layers
}

T. Bánsági Jr. and A. F. Taylor

Citation: Chaos 25, 064308 (2015); doi: 10.1063/1.4921767

View online: http://dx.doi.org/10.1063/1.4921767

View Table of Contents: http://aip.scitation.org/toc/cha/25/6

Published by the American Institute of Physics 


\title{
Helical Turing patterns in the Lengyel-Epstein model in thin cylindrical layers
}

\author{
T. Bánsági, Jr. and A. F. Taylor ${ }^{a)}$ \\ Department of Chemical and Biological Engineering, University of Sheffield, Mappin Street, Sheffield, S1 3JD, \\ United Kingdom
}

(Received 27 March 2015; accepted 11 May 2015; published online 3 June 2015)

\begin{abstract}
The formation of Turing patterns was investigated in thin cylindrical layers using the Lengyel-Epstein model of the chlorine dioxide-iodine-malonic acid reaction. The influence of the width of the layer $\mathrm{W}$ and the diameter $\mathrm{D}$ of the inner cylinder on the pattern with intrinsic wavelength $l$ were determined in simulations with initial random noise perturbations to the uniform state for $\mathrm{W}<l / 2$ and $\mathrm{D} \sim l$ or lower. We show that the geometric constraints of the reaction domain may result in the formation of helical Turing patterns with parameters that give stripes $(b=0.2)$ or spots $(b=0.37)$ in two dimensions. For $b=0.2$, the helices were composed of lamellae and defects were likely as the diameter of the cylinder increased. With $b=0.37$, the helices consisted of semi-cylinders and the orientation of stripes on the outer surface (and hence winding number) increased with increasing diameter until a new stripe appeared. (C) 2015 Author(s). All article content, except where otherwise noted, is licensed under a Creative Commons Attribution 3.0 Unported License.
\end{abstract}

[http://dx.doi.org/10.1063/1.4921767]

In 1952, Turing proposed a general mechanism for biological pattern formation involving the reaction and diffusion of chemical species. Whilst evidence of Turing's mechanism was difficult to obtain in living systems, chemists found hexagonal and striped Turing patterns in the chlorite-iodide-malonic acid (and related CDIMA) reaction in gels in open reactors. Much of the experimental work has focused on quasi-2D chemical patterns. Three-dimensional Turing patterns can be challenging to characterize and thus remain underexplored. Here, we investigate patterns in thin cylindrical layers using the Lengyel-Epstein model of the chlorine dioxide-iodinemalonic acid (CDIMA) reaction. We find that, even in these thin layers, pattern selection can be influenced by the third dimension: helices were obtained from random initial conditions in cylinders when spots were observed in two dimensions. Nevertheless, robust sequences of patterns occurred with increasing domain size.

\section{INTRODUCTION}

In his 1952 paper on the Chemical Basis of Morphogenesis, ${ }^{1}$ Turing proposed that reaction and diffusion of chemical species may account for the appearance of patterns in biological systems. He considered the case of two chemical species, morphogens $\mathrm{X}$ and $\mathrm{Y}$, in a ring of cells; $\mathrm{Y}$ catalyzed its own production and $\mathrm{X}$ had a larger diffusion constant than Y. Perturbation of the homogeneous state resulted in the formation of stationary waves on the ring with a "chemical wavelength" related to the reaction rate and diffusion constants. Turing suggested that the waves might provide a chemical prepattern for the formation of whorls of leaves around the stem of a plant or the growth of tentacles

\footnotetext{
a) Author to whom correspondence should be addressed. Electronic mail: A.F.Taylor@sheffield.ac.uk.
}

on hydra, a sea-creature with a hollow tube-like body. Diffusion-driven instabilities were later explored by Meinhardt, ${ }^{2}$ Murray, ${ }^{3}$ and others. The patterns of spots and stripes obtained in theory and simulations often bore striking resemblance to those observed on animals.

Despite the interest that followed in the application of Turing's ideas to pattern formation in nature, almost forty years passed without clear experimental verification of the patterns. Since it was difficult to identify suitable morphogens in living systems, ${ }^{4}$ the most convincing evidence of Turing patterns was first obtained by chemists with the chlorite-iodide-malonic acid (CIMA) reaction in an open reactor containing a rectangular strip of gel. ${ }^{5}$ In this reactor, the chlorite was separated from the malonic acid with these species diffusing into either side of the long edge of the gel along with the rest of the reactants. A starch-like indicator was used to visualize regions of high concentration of iodide: the resultant starch-tri-iodide complex is blue-black. Viewed from above, rows of hexagonally organized spots appeared perpendicular to the feed gradient. Shortly thereafter patterns were obtained in the same reaction in a gel disk reactor viewed from the side (parallel to the feed gradient). ${ }^{6}$ Hexagons, stripes and a mixed state were obtained with changes in the concentrations of reactants.

Lengyel and Epstein ${ }^{7}$ analyzed the mechanism of the reaction and the related CDMIA reaction and were able to reduce it to two key variables, iodide and chlorite, by assuming that chlorine dioxide, iodine and malonic acid concentrations could be taken as effectively constant compared to the large changes in the concentrations of the other two species. The model was amenable to theoretical analysis and explained the appearance of Turing patterns in the experiments in the open gel reactor. Typically the diffusion constants of small ions in solution are similar in magnitude; the reduced diffusion of the activator iodide relative to chlorite arose as a result of the binding of this species to starch, a natural polymer 
which was unable to diffuse in the gel, thus ensuring the conditions necessary for the Turing instability. This revelation led to the proposal of a new approach to the design of Turing patterns in chemical systems involving the reversible binding of the activator to form an immobile complex. ${ }^{8,9}$

The investigation of three-dimensional Turing patterns was pursued in theory. ${ }^{10}$ Logical extension of the $2 \mathrm{D}$ patterns into 3D was obtained, such as a lamellar (lam) type pattern from stripes, a rod-like extension of spots resulting in hexagonally packed cylinders (hpc), and a body centred cubic (bcc) lattice of spots. In fact, many types of pattern are possible in three dimensions and they coexist for some parameters. In simulations of the Lengyel-Epstein model, complex 3D labyrinth structures were observed as well as simple patterns. ${ }^{11,12}$ Predicting which 3D pattern will be selected can be a challenging task.

In experiments with the gel disk reactor, the wavelength of the pattern was $0.2 \mathrm{~mm}$ and the gels were greater than $1 \mathrm{~mm}$ in thickness, nevertheless patterns appeared essentially two-dimensional. Later it was demonstrated that the chemical gradients in the reactor ensured that only a narrow part of the medium was able to support patterns thus resulting in quasi-2D structures. ${ }^{13}$ In systems in which the depth of the gel was gradually increased the images suggested some degree of three-dimensional character and hexagon patterns obtained in thin capillaries fed from either end with the CIMA reactants appeared to fit the bcc structure. ${ }^{14,15}$ However, the 2D projections of 3D structures can be difficult to interpret. Three-dimensional Turing patterns have only recently been reconstructed in experiments exploiting the Belousov-Zhabotinsky (BZ) reaction in microemulsions in capillary tubes. ${ }^{16}$ In this reaction, the activator is trapped in aqueous droplets and the inhibitor, bromine, is able to diffuse at a much faster rate through the oil phase. Optical tomography was used to elucidate the structures such as hexagonally packed cylinders and lamellar patterns. Curved surfaces such as tubes were also obtained in the narrow capillaries. It is well known that the geometry and size of the domain, as well as the boundary and initial conditions, can influence Turing pattern selection. ${ }^{17}$

In this paper, we return to the circular symmetry of Turing's original work and investigate the formation of Turing patterns in the Lengyel-Epstein model of the CDIMA reaction in thin cylindrical layers. The width of the layer and diameter of the cylinder were similar to or below the intrinsic wavelength of the pattern and conditions were sought for which robust three-dimensional patterns were obtained from random initial conditions. Helical Turing patterns were common in this confined reaction domain.

\section{MODEL} form:

The Lengyel-Epstein (LE) model ${ }^{7,8}$ has the following

$$
\begin{aligned}
& \frac{\partial u}{\partial t}=a-u-4 \frac{u v}{1+u^{2}}+\nabla^{2} u \\
& \frac{\partial v}{\partial t}=\sigma\left[b\left(u-\frac{u v}{1+u^{2}}\right)+c \nabla^{2} v\right]
\end{aligned}
$$

where $u$ and $v$ are the dimensionless concentrations of iodide and chlorite ions, and $a, b, c$, and $\sigma$ are dimensionless parameters: $a$ and $b$ are related to the rate constants of the reactions involved, $c=D_{v} / D_{u}$, where $D$ is the diffusion constant of $u$ or $v$ and $\sigma=1+K[\mathrm{~S}]$, where $K$ is the binding constant of iodide to the indicator, e.g., starch and $[\mathrm{S}]$ is the concentration of starch. We took $a=12, \sigma=50$, and $c=1$ throughout and $b=0.2$ or $b=0.37$. Space is scaled by a characteristic rate and diffusion constant such that space (s.u.) $=\left(\mathrm{k}_{2}\left[\mathrm{ClO}_{2}\right] / D\right)^{1 /}$ ${ }^{2} r$, where the units of $r$ depend on $D .{ }^{18}$ With typical values of the parameters in experiments, the scaling factor is of the order $10 \mathrm{~mm}^{-1}$.

This model has been thoroughly analyzed and we only summarize the results here. ${ }^{18,19}$ The steady state of the system depends only on $a: u_{\mathrm{ss}}=a / 5=2.40$ and $v_{\mathrm{ss}}=1+(a / 5)^{2}$ $=6.76$ for the parameters used in this work. In the CDIMA reaction, positive feedback and hence rate acceleration is obtained as a result of the kinetic term which ensures that as $u$ decreases, its rate of removal increases (i.e., $u /\left(1+u^{2}\right)$ decreases with $u$ for $u>1$; this term decreases for $u<1$ thus limiting the rate of removal at low $u$ ). The variable $u$ is referred to as activator as the first diagonal element of the Jacobian matrix of (1) evaluated at the steady state is positive and given by $\left(\left(8 u^{2} v\right) /\left(u^{2}+1\right)^{2}-(4 v) /\left(u^{2}+1\right)-1\right)_{\mathrm{ss}}$ $=1.8$. The other diagonal element is negative and $v$ is referred to as inhibitor. The parameter $\sigma$ multiplies the ratio of diffusion coefficients $c$. With high binding coefficients $\sigma$ may have values up to 1000 , thus ensuring fast diffusion of $v$ relative to the activator. The model shows both Turing and Hopf bifurcations; the bifurcation curves obtained from linear stability analysis are given by

$$
\begin{aligned}
& b_{T}=\frac{c}{5 a}\left(13 a^{2}-4 \sqrt{10} a \sqrt{25+a^{2}}+125\right), \\
& b_{H}=\frac{3 a}{5 \sigma}-\frac{25}{a \sigma} .
\end{aligned}
$$

Below $b_{\mathrm{T}}$ the steady state is unstable to spatially inhomogeneous perturbations and below $b_{\mathrm{H}}$ temporal oscillations occur. With the values of the parameters used here, $b_{\mathrm{H}}=0.102$ and $b_{\mathrm{T}}=0.395$.

In order to provide some insight into the influence of the cylindrical geometry of the reaction domain on Turing patterns, simulations were first performed on 2D rings; 2D squares and 3D slabs (Fig. 1). The width of the domain was always less than the intrinsic wavelength of the pattern. The equations were solved using FlexPDE on a $2 \mathrm{D}$ or $3 \mathrm{D}$ Cartesian adaptive grid with no-flux boundary conditions. Simulations were initiated by perturbing the spatially uniform steady state in $u$ and were terminated typically at 2000 t.u. or after stable patterns had formed. White noise was applied to the initial values of $u$ by the addition of a uniform distribution of pseudo-random numbers in $\left(0,0.01 u_{\mathrm{ss}}\right)$ to $u_{\mathrm{ss}}$. The effect of the amplitude of the noise on pattern selection was not investigated, however, a different initial distribution of $u$ was generated in each run and multiple runs were performed in cases where highly symmetric structures emerged to test for their sensitivity to initial conditions. 

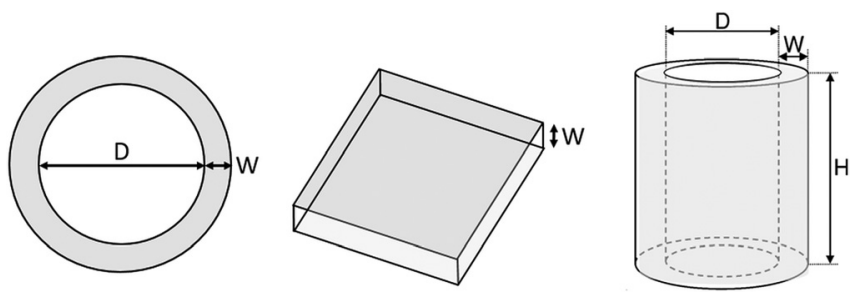

FIG. 1. Reaction domains in simulations: ring, slab and cylindrical layer of inner diameter $\mathrm{D}$, width $\mathrm{W}$, and height $\mathrm{H}$.

\section{RESULTS}

The dispersion relations were obtained from linear stability analysis ${ }^{20}$ and are shown in Figure 2 for the two cases of $b$ explored in this work. The largest growth rate occurred at wave number $\mathrm{k}_{\mathrm{m}}=0.78$ for $b=0.2$ which corresponds to a wavelength of 8.1 s.u. and $\mathrm{k}_{\mathrm{m}}=0.90$ for $b=0.37$ giving a wavelength of 7.0 s.u. On rings of width 0.5 s.u. (essentially) 1D patterns of stripes were obtained (Fig. 2 inset). For $b=0.2$, the band of growing modes was broader and located at lower wave numbers (Fig. 2(a)) compared to the unstable regime for $b=0.37$ (Fig. 2(b)). Consequently, the stable wave configurations on the ring markedly differed: in the former parameter regime the number of waves around the circumference of the ring grew stepwise continuously with ring diameter $\mathrm{D}$, whereas in the latter case a discontinuous trend was observed with increasing ring diameter (Figs. 2(c) and 2(d)). The wavelength on the ring varied from 3.9-9.6 s.u. for $b=0.2$ and 4.8-7.4 s.u. for $b=0.37$ and increased in a sawtooth manner, approaching the wavelength associated with the fastest growing mode for large D.

On rings of larger width with $b=0.37$ semicircles arranged along the perimeter of the ring resulting in the stepwise growth in the number of waves without discontinuity (Figs. 3(a)-3(d)). A circular stripe, brightest at the inner boundary darkest at the outer domain wall, was also observed in approximately half of the runs for all diameters with $\mathrm{D}>0.7$ (Figs. 3(e)-3(g)). A bright stripe at the outer boundary was not favored for these parameters. If an initial
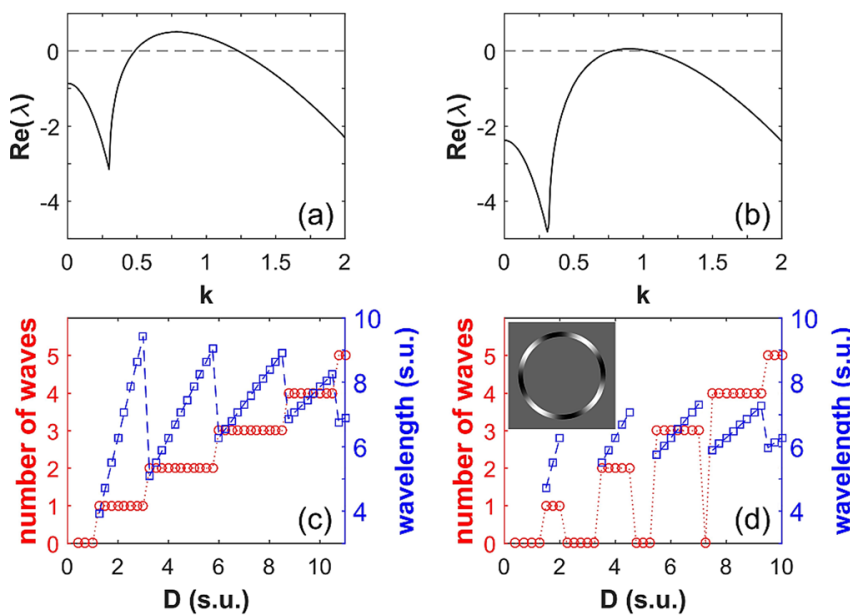

FIG. 2. Dispersion relations as a function of wavenumber $\mathrm{k}$ and number of waves (circles) and wavelength (squares) on a ring of width $\mathrm{W}=0.5$ s.u. in the Lengyel-Epstein model with $b=0.2$ (a, c), $b=0.37$ (b, d), and $a=12$, $\sigma=50$ and $c=1$.

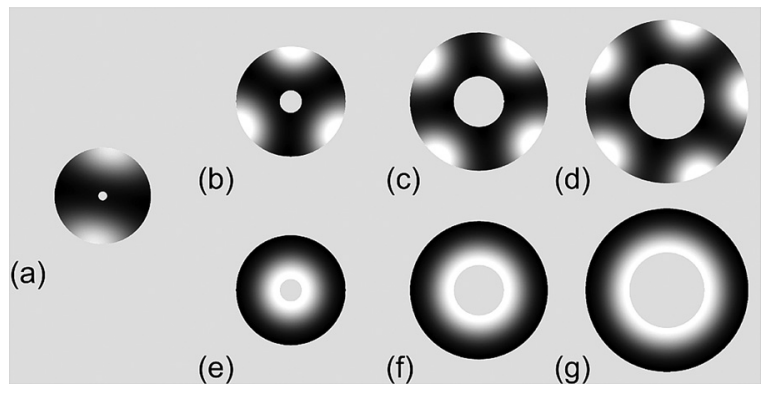

FIG. 3. Turing patterns with $b=0.37$ on a ring of $\mathrm{W}=3.5, \mathrm{D}=0.7$ (a) and coexistence of spotted and striped patterns with $\mathrm{D}=1.75(\mathrm{~b}, \mathrm{e}), 4(\mathrm{c}, \mathrm{f}), 6$ $(\mathrm{d}, \mathrm{g})$. White $=$ low $u$.

condition was imposed such that there was a bright stripe at the outer boundary, it evolved into semicircles with the same configuration as Figs. 3(a)-3(d). With $b=0.2$, the patterns were composed of stripes organized perpendicular to the boundary (not shown).

Two-dimensional simulations on square domains for the chosen parameters yielded familiar labyrinthine and hexagonal Turing patterns (Fig. 4). Allowing the former patterns to expand into the third dimension ( $\mathrm{W}=3.5$ s.u.) preserved symmetry resulting in thin lamellar structures (Fig. 4(b)) indistinguishable from their 2D counterparts. The planar hexagonal Turing patterns responded similarly to the increases in width by forming hexagonally packed cylinders (hpc) in large domains (Fig. 4(d)). Elsewhere, however, long interconnected strands developed. Projection of the overall pattern on the top or bottom of the slab was reminiscent of Turing patterns composed of a mixture of spots and stripes. Closer inspection revealed that the cross section of the strands was in fact a semicircle with the diameter segment attached to the wall and the arc residing inside the layer. Thus, 2D spots formed cylinders or semi-cylinders in 3D.

In cylindrical layers of width $\mathrm{W}<3.5$ s.u. only horizontal stripes were observed for both values of $b$ (Figs. 5(a) and $5(\mathrm{~g}))$ when the diameter was below 0.5 s.u. This common behavior at high curvatures can be interpreted as a result of the Turing instability effectively taking place in one dimension. With $\mathrm{W}=0.5$ s.u., an increase in $\mathrm{D}$ resulted in the reappearance spots for $b=0.37$ (Figs. 5(b)-5(f)) and labyrinth patterns for $b=0.2$ (Figs. 5(h)-5(1)) that extended through

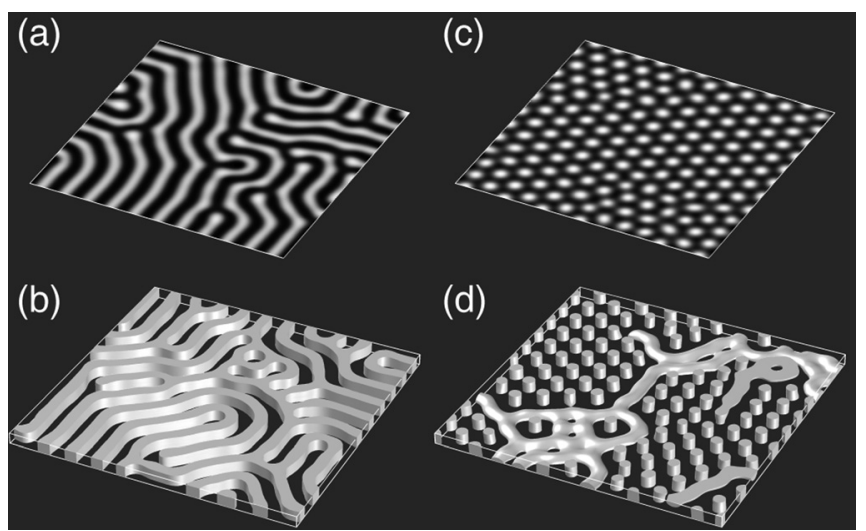

FIG. 4. Turing patterns on a square planar domain $(90 \times 90$ s.u. $)$ and in slabs of width $\mathrm{W}=3.5$ s.u. with $b=0.2(\mathrm{a}, \mathrm{b})$ and $b=0.37$ (c,d). 

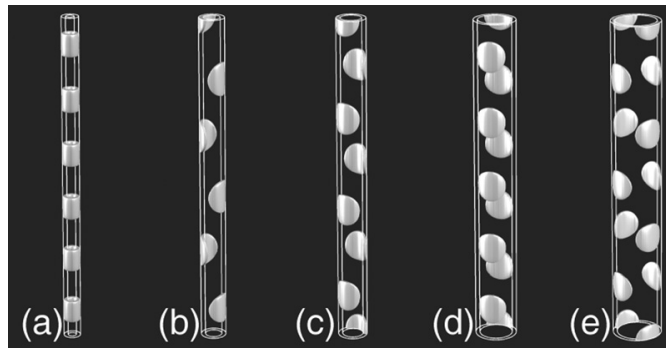

(f)
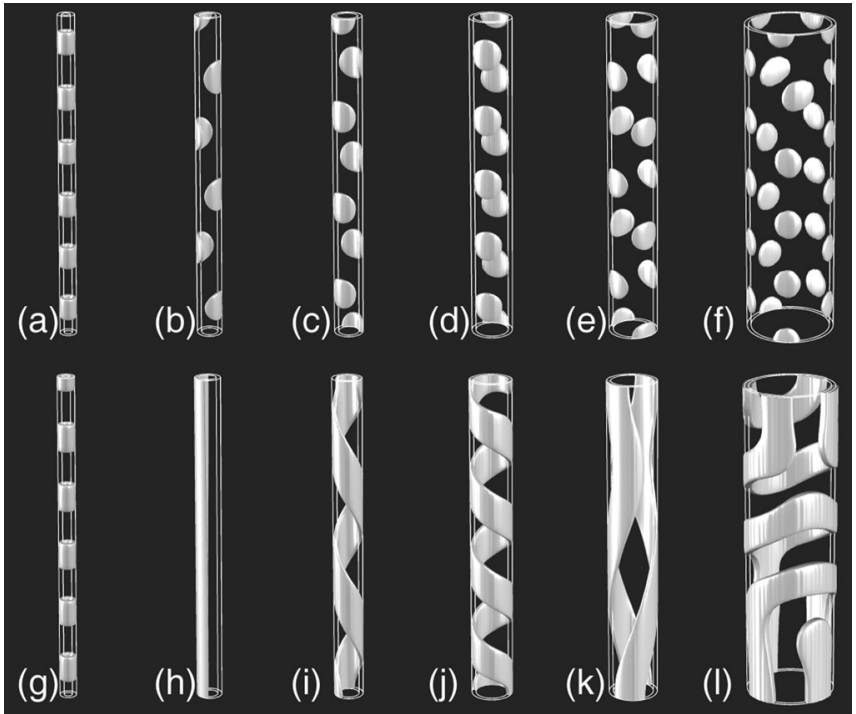

FIG. 5. Turing patterns with $b=0.37$ (a-f) and $b=0.2$ (g-1) in thin cylindrical layers with $\mathrm{W}=0.5$ s.u., $\mathrm{H}=40$ s.u and $\mathrm{D}$ increasing from $\mathrm{D}=1(\mathrm{a}, \mathrm{g}), 2$ (b,h), $2.5(\mathrm{c}, \mathrm{i}), 4(\mathrm{~d}, \mathrm{j}), 5(\mathrm{e}, \mathrm{k}), 10$ (f,l) s.u.

the full width of the domain in both cases. The configuration of spots was found to be dependent on the diameter of the thin cylindrical layer (Appendix). In the striped regime, for small diameters first a single stripe developed (Fig. 5(h)) that started to wind as the curvature decreased (Figs. 5(i) and $5(j))$ until a second stripe appeared. Further domain increase resulted in not only helical winding of the pair of stripes but also a growing number of irregularities, and eventually only labyrinthine patterns developed (Fig. 5(1)). Winding occurred in both directions; examples of left-handed helices are shown here.

Helical patterns were also obtained in the cylindrical layers with $b=0.37$ as the size of the domain was increased. For $\mathrm{W}=1.1$ s.u., the spots on the cylinder (Fig. 6(b)) extended from the outer to the inner boundary. As the width of the layer was increased, conical frusta were observed (Fig. 6(d)) that eventually detached from the inner surface giving semicircles in the cross-section of the cylinder. With a further increase to $\mathrm{W}=3.4$ s.u., a double helix appeared (Fig. 6(f)). Helices did not form if the value of $b$ was increased to 0.39 ; in this case spots were the only stable pattern.

A robust sequence of patterns occurred as the diameter of cylinder was increased with $\mathrm{b}=0.37$ and $\mathrm{W}=3.5$. Short strands perpendicular to the long axis formed at low

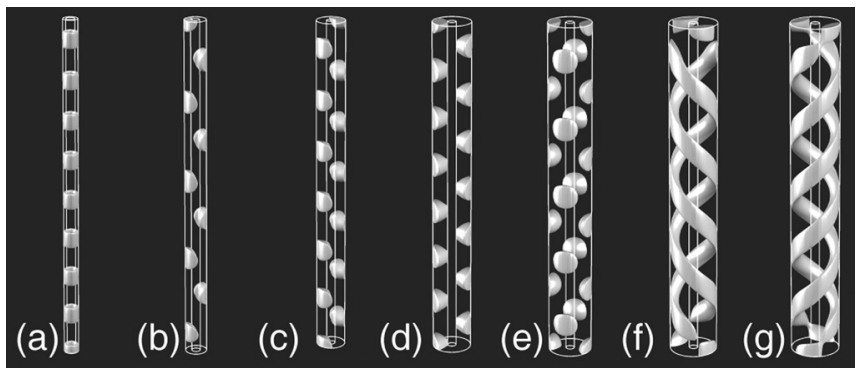

FIG. 6. Turing patterns with $b=0.37, \mathrm{D}=1.5$ s.u., $\mathrm{H}=60$ s.u., and $\mathrm{W}=0.5$ (a), 1.1 (b), 1.75 (c), 2.6 (d), 3.2 (e), 3.4 (f), 3.5 (g) s.u.
D (Fig. 7(a)). With increasing diameter the segments rearranged into the structure seen in Fig. 5(b) and subsequently into a double helix (Fig. 7(c)). Further increasing the diameter brought about an additional strand arranging the pattern into a triple helix (Figs. 7(d) and 7(e)) before a highly symmetric assembly of inwardly tapered conical frusta formed (Fig. 7(f)). At this point the increasing diameter favored again strand formation (Figs. 7(g) and 7(h)) and conical frusta gave way until they once more gained stability over strands (Fig. 7(i)). This sequence of competition between the two markedly different arrangements continued, this time involving five strands before eventually conical frusta established dominance.

Tiles of the patterns obtained from unfolding the outer face of the cylinder as D was increased between 2 s.u. (three vertical stripes) and 4.4 s.u. (four vertical stripes) are shown in Figures 8(a)-8(1). The rotated striped pattern associated with the helices occurred for most values of $\mathrm{D}$ except for $\mathrm{D}=3.6-3.9$ where the spotted pattern emerged. The crosssection of the cylindrical layer revealed a striped pattern when spots were obtained on the tile (Fig. $8(\mathrm{~m})$ ) whereas a pattern of semicircles (Figs. 8(n) and 8(o)) occurred when the tile was striped. In this confined domain, only one of the two structures observed in the 3D slab, either semi-cylinders or cylinders, was selected. The orientation of the stripes relative to the vertical was governed by the diameter of the cylinder and increased from 0 to $\pi / 4$ before returning to 0 again when four vertical stripes developed.

For parameters producing labyrinthine patterns in 2D, the increase in layer width to $W=3.5$ s.u. induced no symmetry change at small diameters, stripes only grew laterally (Fig. 9(a)). As the domain became larger a single helix emerged initially which in fact was the only robust symmetric 3D structure (Fig. 9(b)). With increasing diameter, all subsequent patterns were combinations of short symmetric single-and double helical as well as stacked band segments

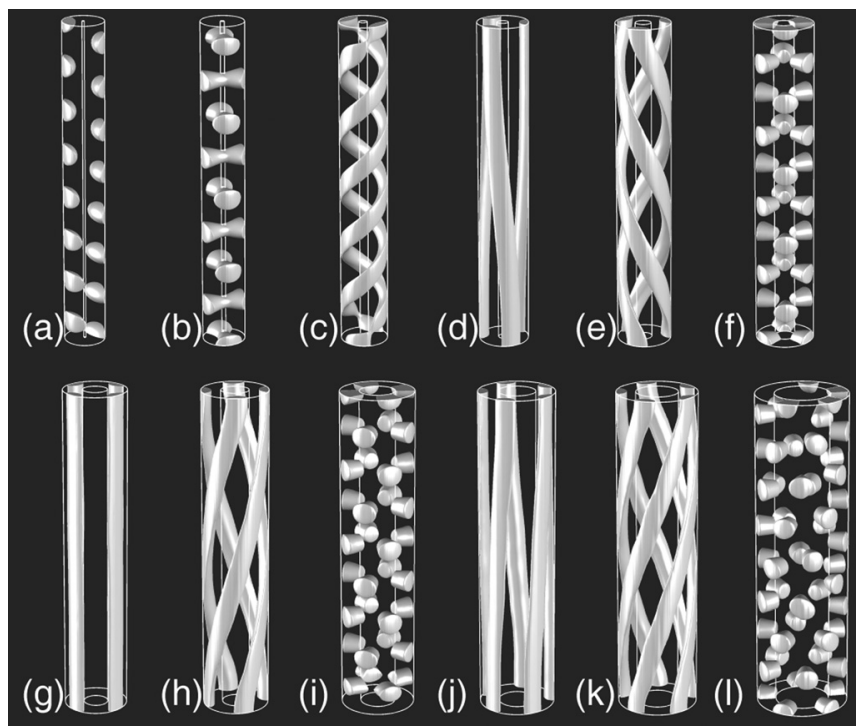

FIG. 7. Turing patterns with $b=0.37, \mathrm{~W}=3.5$ s.u., $\mathrm{H}=60$ s.u., and $\mathrm{D}=0.4$ (a), 0.7 (b), 1.5 (c), 2 (d), 2.8 (e), 3.6 (f), 4.5 (g), 5 (h), 6.4 (i), 6.8 (j), 7.6 (k), 10 (l) s.u. 


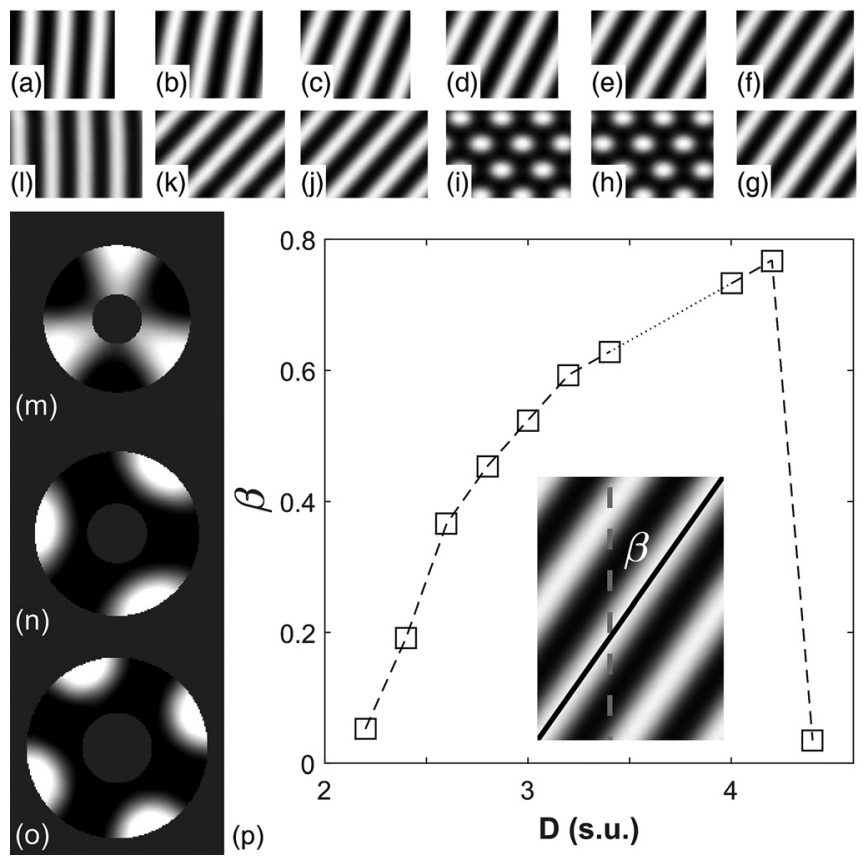

FIG. 8. Tiles $(\sim 20 \times 20$ s.u. $)$ constructed from unfolding the outer face of the cylindrical layers in Fig. 8 with increasing D (a-1). Cross-sections of the cylindrical layers in $(\mathrm{h}),(\mathrm{j})$, and $(\mathrm{l})$, are shown in $(\mathrm{m}),(\mathrm{n})$, and $(\mathrm{o})$ for $\mathrm{D}=3.6,4.0$, and 4.4 s.u., respectively. (p) Angle $\beta$ of stripes plotted as a function of $\mathrm{D}$ (dotted line corresponds to spotted patterns).

(Figs. 9(c)-9(f)) until eventually labyrinthine Turing patterns prevailed.

\section{DISCUSSION AND CONCLUSIONS}

The LE model ${ }^{7}$ was introduced to explain Turing patterns in the CIMA or related CDMIA reaction in open gel reactors in the early 1990s. ${ }^{5,6}$ In Turing's analysis ${ }^{1}$ stationary patterns arose through a combination of autocatalysis and differential diffusion. Lengyel and Epstein demonstrated that Turing patterns can be obtained in a model where positive feedback arises through the rate of removal of a reactant and differential transport occurs through binding of the activator to a large, immobile, species. Here, we investigated Turing patterns in the LE model returning to the circular symmetry of Turing's original paper in order to elucidate the possible three dimensional structures arising with this geometric constraint.

Pattern selection is a complex problem studied theoretically in amplitude equations. ${ }^{20}$ The Brusselator model was more frequently employed in investigations than the LE; the

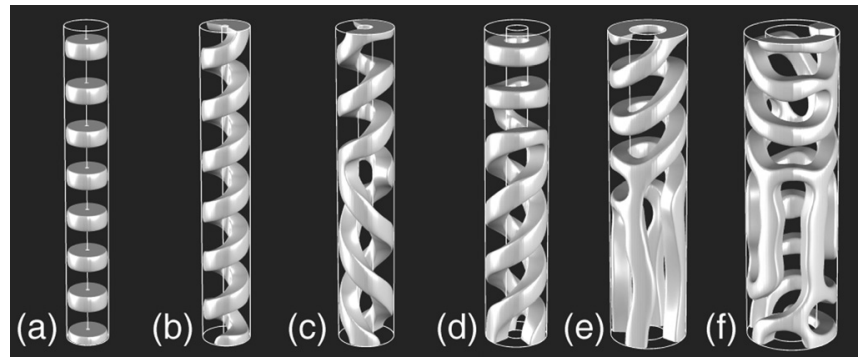

FIG. 9. Turing patterns with $b=0.2, \mathrm{~W}=3.5$ s.u., $\mathrm{H}=60$ s.u., and $\mathrm{D}=0.4$ (a), 1.5 (b), 3 (c), 4 (d), 7 (e), 10 (f) s.u. two have been compared in some cases and display common features. ${ }^{21,22}$ Many pattern types are possible in two and three dimensions and multistability between patterns is exhibited. Prediction of the type of pattern selected is therefore a formidable task. In the LE, stripes and two types of hexagonal spot patterns are produced in two dimensions. ${ }^{18,23}$ The simplest three-dimensional patterns are extensions of the 2D structures: lamellae (lam) from stripes, the body center cubic lattice of spheres from spots (bcc) and hexagonal packed cylinders which are spots in one plane and stripes in the other (hpc), however, other structures have been obtained in simulations. ${ }^{24,25}$ Typically regular patterns appear over small regions of space with dislocations and defects occurring in larger domains. Simulations in large 3D domains yielded complex structures that were difficult to characterize. $^{12}$

Here, patterns were compared in simulations of the LE for two different values of the parameter $b$. The dispersion relation with $b=0.2$ resulted in a broad band of unstable modes, whereas $b=0.37$ had a narrow band. Hence, the number of waves on a thin $2 \mathrm{D}$ ring $(\mathrm{W}=0.5)$ grew as a stepwise continuous function of the ring diameter for $b=0.2$ whilst discontinuities were obtained for $b=0.37$ : a narrower band of unstable modes reduced the flexibility in wavelength selection required to overcome domain length constraints. As the diameter of the ring was increased, the intrinsic wavelength $l$ associated with the maximum growth rate of the pattern was approached.

In square domains, hexagons were obtained for $b=0.37$ from random initial conditions. In $2 \mathrm{D}$ rings $(\mathrm{W}=3.5)$, co-existence was observed between a semicircle attached to the outer boundary and striped pattern. Cylinders or semicylinders appeared in slabs, resulting in hpc in some parts of the domain and semi-cylinders in other parts, i.e., a mixed spot/stripe pattern in the $2 \mathrm{D}$ projection. With $b=0.2$, stripes were obtained on $2 \mathrm{D}$ rings and in slabs the patterns preserved their 2D symmetry resulting in lamellar labyrinth patterns.

Confining the reaction in the cylindrical layer resulted in robust three-dimensional helical patterns for some parameters. Observed from the outer surface, the patterns took the form of stripes that wound around the cylinder. Helices arose as a result of the coupling between the constraint of the wavelength selection on the cross-sectional ring and the greater freedom on the surface of the cylinder. As the ring increased in diameter, only certain unstable modes were able to grow on the ring and satisfy the periodic boundary conditions whereas the fastest growing mode could still be obtained on the surface by rotation of the striped structure. Hence, the orientation of stripes (and hence winding number of the helices) depended on the diameter of the inner cylinder.

The major difference between helices for the two values of $b$ arose from comparison of the three-dimensional nature of the pattern. With $b=0.2$, the patterns extended through the width of the layer with axial symmetry. With $b=0.37$, cross-sections revealed that the helices were semi-cylinders attached to the outer surface. In our investigations, reaction domains of width $\mathrm{W}<l / 2$ and diameter $\sim l$ or lower were employed; even in these small domains, defects and 
combinations of structures were observed. For both values of $b$, as the size of the domain grew then the probability of defects increased, however, the helices were obtained in robust sequences with $b=0.37$ suggesting that the narrower band of unstable modes observed in the dispersion curve restricted pattern selection.

The LE model reproduces many experimental features including the light sensitivity of the reaction; new structures such as superlattice patterns have been obtained by forcing and coupling of gel layers. ${ }^{26-29}$ Most of the experimental patterns are essentially $2 \mathrm{D}$ in nature and in cases where patterns are $3 \mathrm{D}$, elucidation of structures from $2 \mathrm{D}$ projections can be challenging. The reaction does not lend itself to the techniques that have been exploited with the $\mathrm{BZ}$ reaction such as optical tomography and MRI. ${ }^{30}$ Patterns are easier to analyze in thin layers but can still be affected by threedimensional effects.

Helical structures are common on the nano- to microscale in crystalline chemical systems and the combination of reaction and diffusion sometimes plays a role in their formation. ${ }^{31}$ Examples of helical patterns arising from the interplay of autocatalytic chemical reaction and differential transport have not been illustrated in experiments. The boundary conditions used in this work do not apply to the open gel reactors; however, helical patterns may still be obtained in cylindrical layers with a boundary feed from a CSTR. $^{32}$ When 3D Turing patterns were reconstructed from the BZ microemulsion in capillaries, semi-spheres, and semi-cylinders were observed attached to the outer boundary. Conditions might be exploited for which transient Turing patterns were produced in the CDIMA reaction in closed reactors and the reaction performed in the annulus between two capillaries in order to obtain the results of the simulations presented here. ${ }^{14,33}$

One of the remarkable features of Turing's work is that the underlying mechanism of positive feedback and differential transport has been applied to explain a wide range of natural patterns occurring on vastly different length and time scales, from inorganic precipitates to flames, vegetation and populations of animals. ${ }^{34-36}$ From a biological perspective, simulations have provided much insight into the role a Turing instability might play in, for example, animal markings. ${ }^{37,38}$ Problems such as the lack of robustness of Turing patterns continue to be an issue but have been tackled through various strategies such as a slowly growing domain. ${ }^{39}$ Turing patterns have been investigated on spherical and hemispherical surfaces ${ }^{40}$ but not, to our knowledge, in thin cylindrical layers before. It seems unlikely that the results reported here are confined to the LE model of the CDIMA reaction. Thus, helical structures might be obtained in other systems where Turing patterns arise.

\section{ACKNOWLEDGMENTS}

The authors acknowledge Grant No. EPSRC EP/ K030574/1 and Marie Curie International Incoming Fellowship (PIIF-GA-2010-274677). We dedicate this paper to Professor Irving Epstein on the occasion of his 70th birthday.

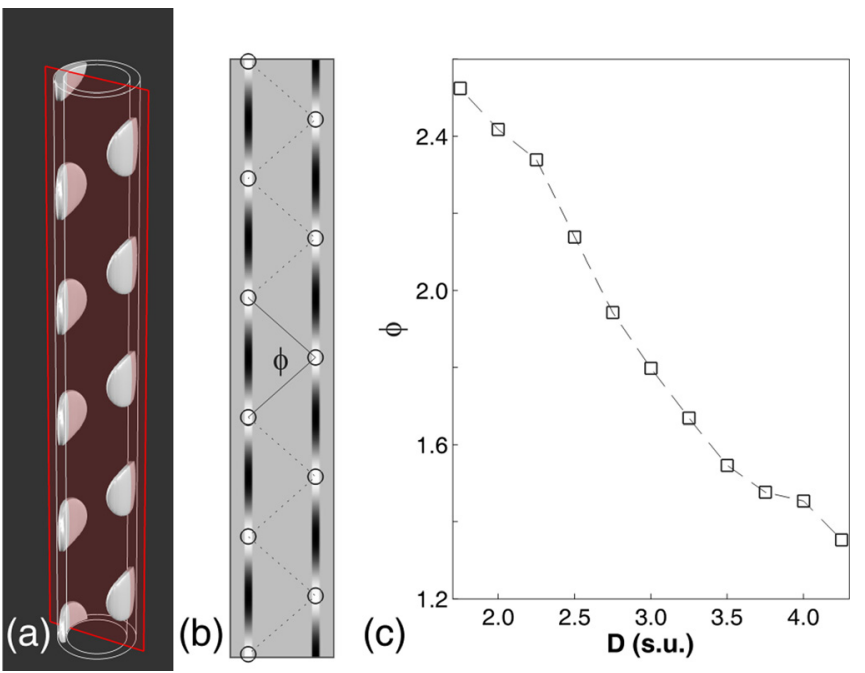

FIG. 10. Configuration of spotted Turing patterns with $b=0.37, \mathrm{~W}=0.5$ s.u., $H=40$ s.u., and $\mathrm{D}=4$ s.u. (a,b). The angle between the center of spots shown in (b) is plotted as a function of D in (c).

\section{APPENDIX: CONFIGURATION OF SPOTS ON CYLINDERS}

The angle, $\phi$, between the center of spots (blue circles, Fig. 10(b)) measured in a plane cutting through their center (red rectangle, Figs. 10(a) and 10(b)) became smaller as the domain grew until the spots switched to the next stable configuration about $\mathrm{D}=4.3$. In the diameter regime $4.5<\mathrm{D}<5.5$ spots were arranged in twos, one opposite the other with each pair rotated by $\pi / 2$ with respect to the pair below. Further increase in diameter gave rise to similar configurations where three, four, five, etc. spots resided at one height rotated by $\pi / n$ with respect to the next ring of spots up or down the layer, $n$ being the number spots on a ring. Lessordered arrangements containing grain boundaries started to develop and become more frequent as the diameter grew.

\footnotetext{
${ }^{1}$ A. M. Turing, Philos. Trans. R. Soc. London, Ser. B 237, 37 (1952).

${ }^{2}$ H. Meinhardt, Models of Biological Pattern Formation (Academic Press, New York, 1982).

${ }^{3}$ J. D. Murray, Mathematical Biology (Springer, Berlin, 1989).

${ }^{4}$ P. K. Maini, R. E. Baker, and C. M. Chuong, Science 314, 1397 (2006).

${ }^{5}$ V. Castets, E. Dulos, J. Boissonade, and P. Dekepper, Phys. Rev. Lett. 64, 2953 (1990)

${ }^{6}$ Q. Ouyang and H. L. Swinney, Nature 352, 610 (1991).

${ }^{7}$ I. Lengyel and I. R. Epstein, Science 251, 650 (1991).

${ }^{8}$ I. Lengyel and I. R. Epstein, Proc. Natl. Acad. Sci. U.S.A. 89, 3977 (1992).

${ }^{9}$ J. Horvath, I. Szalai, and P. De Kepper, Science 324, 772 (2009).

${ }^{10}$ A. DeWit, P. Borckmans, and G. Dewel, Proc. Natl. Acad. Sci. U.S.A. 94, 12765 (1997).

${ }^{11}$ P. K. Moore and W. Horsthemke, Chaos 19, 043116 (2009).

${ }^{12}$ T. Leppanen, M. Karttunen, K. Kaski, and R. A. Barrio, Int. J. Mod. Phys. B 17, 5541 (2003).

${ }^{13}$ I. Lengyel, S. Kadar, and I. R. Epstein, Phys. Rev. Lett. 69, 2729 (1992).

${ }^{14}$ K. Agladze, E. Dulos, and P. Dekepper, J. Phys. Chem. 96, 2400 (1992).

${ }^{15}$ E. Dulos, P. Davies, B. Rudovics, and P. DeKepper, Physica D 98, 53 (1996).

${ }^{16}$ T. Bánsági, Jr., V. K. Vanag, and I. R. Epstein, Science 331, 1309 (2011).

${ }^{17}$ P. Arcuri and J. D. Murray, J. Math. Biol. 24, 141 (1986).

${ }^{18}$ B. Rudovics, E. Barillot, P. W. Davies, E. Dulos, J. Boissonade, and P. De Kepper, J. Phys. Chem. A 103, 1790 (1999).

${ }^{19}$ J. A. Pojman and I. R. Epstein, An Introduction to Nonlinear Chemical Dynamics (Oxford University Press, New York, 1998).
} 
${ }^{20} \mathrm{M}$. Cross and H. Greenside, Pattern Formation and Dynamics in Nonequilibrium Systems (Cambridge University Press, New York, 2009).

${ }^{21}$ P. Borckmans, A. Dewit, and G. Dewel, Physica A 188, 137 (1992).

${ }^{22}$ T. K. Callahan and E. Knobloch, Physica D 132, 339 (1999).

${ }^{23}$ A. Rovinsky and M. Menzinger, Phys. Rev. A 46, 6315 (1992).

${ }^{24}$ A. Dewit, G. Dewel, P. Borckmans, and D. Walgraef, Physica D 61, 289 (1992).

${ }^{25}$ H. Shoji, K. Yamada, D. Ueyama, and T. Ohta, Phys. Rev. E 75, 13 (2007).

${ }^{26}$ I. Berenstein, L. Yang, M. Dolnik, A. M. Zhabotinsky, and I. R. Epstein, Phys. Rev. Lett. 91, 4 (2003).

${ }^{27}$ D. G. Miguez, M. Dolnik, I. Epstein, and A. P. Munuzuri, Phys. Rev. E 84, 6 (2011).

${ }^{28}$ D. Feldman, R. Nagao, T. Bánsági, Jr., I. R. Epstein, and M. Dolnik, Phys. Chem. Chem. Phys. 14, 6577 (2012).

${ }^{29}$ J. Li, H. L. Wang, and Q. Ouyang, Chaos 24, 023115 (2014).
${ }^{30}$ M. M. Britton, A. J. Sederman, A. F. Taylor, S. K. Scott, and L. F. Gladden, J. Phys. Chem. A 109, 8306 (2005).

${ }^{31}$ S. Thomas, I. Lagzi, F. Molnar, and Z. Racz, Phys. Rev. Lett. 110, 5 (2013).

${ }^{32}$ D. E. Strier, P. de Kepper, and J. Boissonade, J. Phys. Chem. A 109, 1357 (2005).

${ }^{33}$ I. Lengyel, S. Kadar, and I. R. Epstein, Science 259, 493 (1993).

${ }^{34}$ V. Petrov, S. K. Scott, and K. Showalter, Philos. Trans. R. Soc. London, Ser. A 347, 631 (1994).

${ }^{35}$ S. C. Muller and J. Ross, J. Phys. Chem. A 107, 7997 (2003).

${ }^{36}$ M. Baurmann, T. Gross, and U. Feudel, J. Theor. Biol. 245, 220 (2007).

${ }^{37}$ S. Kondo and T. Miura, Science 329, 1616 (2010).

${ }^{38}$ J. D. Murray, J. Theor. Biol. 88, 161 (1981).

${ }^{39}$ P. K. Maini, T. E. Woolley, R. E. Baker, E. A. Gaffney, and S. S. Lee, Interface Focus 2, 487 (2012).

${ }^{40}$ C. Varea, J. L. Aragon, and R. A. Barrio, Phys. Rev. E 60, 4588 (1999). 